\title{
Dynamic SU(2) Lattice Gauge Theory at Finite Temperature*
}

\author{
K. Okano* ${ }^{\dagger}$, L. Schülke and B. Zheng \\ Universität - GH Siegen, D - 57068 Siegen, Germany \\ * University of California Los Angeles, CA90095-1547, US
}

\begin{abstract}
The dynamic relaxation process for the $(2+1)$-dimensional $\mathrm{SU}(2)$ lattice gauge theory at critical temperature is investigated with Monte Carlo methods. The critical initial increase of the Polyakov loop is observed. The dynamic exponents $\theta$ and $z$ as well as the static critical exponent $\beta / \nu$ are determined from the power law behaviour of the Polyakov loop, the autocorrelation and the second moment at the early stage of the time evolution. The results are well consistent and universal short-time scaling behaviour of the dynamic system is confirmed. The values of the exponents show that the dynamic $\mathrm{SU}(2)$ lattice gauge theory is in the same dynamic universality class as the dynamic Ising model.
\end{abstract}

PACS: 11.15.Ha, 11.10.Wx, 02.60.Cb, 05.70.Jk

${ }^{*}$ Work supported in part by the Deutsche Forschungsgemeinschaft; DFG Schu 95/9-1

${ }^{\dagger}$ On leave of absence from Tokuyama University, Tokuyama-shi, Yamaguchi 754, Japan 
In recent years lattice gauge theory has continuously been developing. Numerical simulations have been extended to the gauge theory with fermions and at finite temperature. Up to now, however, most of simulations are devoted to the equilibrium state. Non-equilibrium processes have not been studied so deeply. In this letter, we report the first numerical simulation of the short-time dynamics for the $(2+1)$-dimensional $\mathrm{SU}(2)$ lattice gauge theory at the critical temperature.

Recently great progress has been achieved in critical dynamics for spin systems. For long it was believed that no universal behaviour would be present in the short-time regime of critical dynamics. However, for the critical relaxation process starting from an initial state with very high temperature and small magnetization, it was recently argued by Janssen, Schaub and Schmittmann [1] with renormalization group methods that there exist universality and scaling even at macroscopic early times, which sets in right after a microscopic time scale $t_{m i c}$. Based on the scaling relation it was predicted that at the beginning of the time evolution the magnetization surprisingly undergoes a critical initial increase

$$
M(t) \sim m_{0} t^{\theta}
$$

where $\theta$ is a new dynamic exponent.

Numerical simulations support the above predictions. The critical initial increase of the magnetization in Eq. (11) was observed for the Ising model and the Potts model and the exponent $\theta$ was directly measured [2, 3]. The scaling relation and universality are confirmed 4.,5,2, 6, 8]. The microscopic time scale $t_{m i c}$ is around 5-60 Monte Carlo time steps, depending on the observables and the microscopic details. If one argues that a Monte Carlo time step is a typical microscopic time unit, such a result is reasonable. Compared with in the long-time regime, the scaling variable $t$ in short-time dynamics plays a more important role in understanding the scaling behaviour, especially in numerical simulations. The investigation of the universal behaviour of the short-time dynamics not only enlarges the fundamental knowledge on critical phenomena but also, more interestingly, provides possible new ways to determine all the dynamic exponents as well as the static exponents from the short-time dynamics, either based on the power law behaviour of the observables at the beginning of the time evolution [3, 9, 1, 10], or on finite size scaling [11, 12]. An appealing feature of these methods is that they may be free of critical slowing down

At this stage, it is natural to ask whether such an investigation may be generalized to field theory. This would be very important for the understanding of the non-equilibrium properties of field theory and numerical simulations of lattice gauge theory. On the other hand, the two-dimensional Ising and 3-state Potts model are known to be the simplest models presenting critical phenomena. They are known to have quite clean behaviour in many respects. One may wonder whether nice universal short-time behaviour is special for these simple systems. For example, it could be that the microscopic time scale $t_{\text {mic }}$ for more complicated systems is so big such that it is comparable with the macroscopic time scale. Then we can not observe any universal behaviour in the macroscopic short-time regime.

As a first approach to the dynamic gauge theory, we will numerically investigate the $(2+1)$-dimensional $\mathrm{SU}(2)$ lattice gauge theory at finite temperature with a dynamics of model A [13] even though there exists so far not any analytical investigation. The motivation to choose this model is that the deconfining phase transition observed in this model is the second order. On the other hand, it is expected that in equilibrium the $(2+1)$-dimensional 
$\mathrm{SU}(2)$ lattice gauge theory is in the same universality class as the two-dimensional Ising model [14]. This has also been numerically observed [15, 16]. It is interesting to investigate whether the dynamic $\mathrm{SU}(2)$ lattice gauge theory is also in the same dynamic universality class as the dynamic Ising model.

The (2+1)-dimensional SU(2) lattice gauge theory is described by the Hamiltonian

$$
H=-\frac{4}{g^{2}} \sum_{P} U_{P}
$$

where the index $P$ indicates the sum over all the fundamental plaquettes

$$
U_{P}=U_{\mu}(x) U_{\nu}(x+\hat{\mu}) U_{\mu}^{\dagger}(x+\hat{\mu}+\hat{\nu}) U_{\nu}^{\dagger}(x+\hat{\nu})
$$

with $\mu, \nu$ denoting the directions in the $(2+1)$ dimensional space. As a finite temperature theory, the lattice size should be taken to be $N^{2} \times N_{0}$. Here $N_{0}$ corresponds to the inverse temperature. Since the theory is super-renormalizable, the continuum scaling law is of the simple form $N_{0} T \sim g^{-2}$. For this $\mathrm{SU}(2)$ lattice gauge theory in equilibrium there exist already rather good numerical results. For a lattice with $N=64$ and $N_{0}=2$, Christensen and Damgaard obtained the critical point $4 / g_{c}^{2}=3.39$ and the exponent $\beta=0.120(8)$ [15], while Teper got the critical point $4 / g_{c}^{2}=3.47$ and the exponent $\nu=0.98(4)$ [16]. Compared with the exact values $\beta=0.125$ and $\nu=1.0$ for the two-dimensional Ising model, the numerical results of the critical exponents for the $\mathrm{SU}(2)$ lattice gauge theory support that both models are in the same universality class. Values for the critical point from the two groups of authors show some small difference. The determination of the critical point to a very rigorous level is at the present stage still difficult. For simplicity, in this paper we will take the simple average $4 / g_{c}^{2}=3.43$ of the above given two values as an input. Our numerical results show that this value for the critical point is very close to the real one.

In order to simulate a critical relaxation, one should first prepare an initial state. The magnetization in the $\mathrm{SU}(2)$ lattice gauge theory is defined as the globally averaged Polyakov loop

$$
M(t)=\frac{1}{N^{2}} \sum_{i}<W_{i}(t)>
$$

where $W_{i}$ is the Polyakov loop at site $i$ which locates in the two-dimensional lattice with lattice size $N^{2}$. The average $\langle\ldots\rangle$ is over the random forces and the independent initial configurations. Compared with the Ising model the Polyakov loop $W_{i}$ plays the role of the Ising spin $S_{i}$ ]. Following the idea of Janssen, Schaub and Schmittmann [1], the initial state should have zero spatial correlation length and small initial magnetization. To get initial configurations with zero spatial correlation, we just remember that for fixed $N_{0}$, the center symmetry $Z_{2}$ of the $\mathrm{SU}(2)$ theory is broken for large $4 / g^{2}$, while it remains unbroken

\footnotetext{
${ }^{1}$ However, $W_{i}$ in the $\mathrm{SU}(2)$ theory is a real variable defined in the interval $[-1,1]$ while the spin $S_{i}$ takes integers \pm 1 . Therefore the $\mathrm{SU}(2)$ lattice gauge theory should contain more physical contents. But in this paper we will not discuss this in details.
} 
for small $4 / g^{2}$. Therefore, for the initial state we take the coupling $4 / g^{2}=0$. A nonzero initial magnetization can be achieved in many ways. A natural one is to introduce an initial external magnetic field $h$. Summarizing, the initial configurations can be generated by the initial Hamiltonian $H_{0}=h \sum_{i} W_{i}$. Final procedure is to adjust the configuration so generated as to give the initial magnetization sharply. Different methods can be found for this sharp preparation in literature in statistical physics [2, 3, 0, 8, 17, 18].

After an initial configuration is prepared, the system is suddenly quenched to the critical temperature with the Hamiltonian in Eq. (2) and then released to evolve with a dynamics of model A. In this paper we adopt the heat-bath algorithm for the dynamic evolution. We stop the update of the dynamic system at a reasonable time, which is typically some hundreds Monte Carlo time steps, and repeat the process. The average is taken over different independent initial configurations and random numbers. The total sample for the lattice size up to $N=64$ is 9600 while for $N=128$ is 8000 . Errors are estimated by dividing the total sample into three or five groups.

In Fig. 1, time evolution of the magnetization is plotted in double-log scale for different initial magnetization $m_{0}$ and lattice sizes $N$. The solid line above is the magnetization profile for $m_{0}=0.04$ and $N=64$, while the dotted and dashed line are those for $N=16$ and 32 respectively. The solid line below is the time dependent magnetization with $m_{0}=0.02$ and $N=64$. From the figure one can realize that in the first Monte Carlo time step the magnetization drops. For example, for $M(t=0)=m_{0}=0.04$ with lattice size $N=64$ it drops to $M(t=1)=0.026$. Such a dropping within the microscopic time scale $t_{m i c}$ is a typical non-universal behaviour which essentially depends on the microscopic details. Similar phenomena have also been observed for the two-dimensional Potts model and the XY model [7,8]. With the Metropolis algorithm for the Potts model, the magnetization decreases continuously even up to around 10 Monte Carlo time steps. From Fig. 1, we clearly see that after one Monte Carlo time step, the magnetization indeed increases and for big enough lattice sizes it is quickly stabilized to the universal power law behaviour given in Eq. (11). The microscopic time scale is $t_{m i c} \sim 20$. For $m_{0}=0.04$, the magnetization profiles for $N=32$ and 64 do not show a big difference. The finite size effect for $N=64$ is already quite small. From the slope of the curves in Fig. 1, one can measure the critical exponent $\theta$. For $m_{0}=0.04$ and $N=64$, from a time interval [20,250] we obtain the exponent $\theta=0.192(2)$. This value is very well consistent with that for the two dimensional Ising model [7].9]. The comparison can be done in Table [1. In Table [1], the value of the exponent $\beta / \nu$ for the Ising model is exact while those of the exponents $\theta$ and $z$ are taken from the literature [7]. Rigorously speaking, the critical exponent $\theta$ is defined in the limit $m_{0}=0$. A numerical measurement in this limit is practically not possible. Therefore, a linear extrapolation to the limit $m_{0}=0$ from finite $m_{0}$ should in principle be carried out [3, ]. For this reason, we have also performed the simulation for $m_{0}=0.02$ and $N=64$. Since $m_{0}$ now is smaller, the fluctuations become bigger. The measured exponent is $\theta=0.186(12)$. Within the errors we can not distinguish the results for $m_{0}=0.02$ and $m_{0}=0.04$. Therefore in this paper a linear extrapolation will not be performed.

Now we set $m_{0}=0$ and proceed to measure the auto-correlation $A(t)$ and the second moment $M^{(2)}(t)$. From a careful scaling analysis for Ising-like systems [19,1], we expect the short-time dynamic scaling behaviours 


$$
\begin{gathered}
A(t) \equiv \frac{1}{N^{2}} \sum_{i}<W_{i}(t) W_{i}(0)>\sim t^{\theta-d / z} \\
M^{(2)}(t) \equiv \frac{1}{N^{4}}<\left(\sum_{i} W_{i}(t)\right)^{2}>\sim t^{(d-2 \beta / \nu) / z}
\end{gathered}
$$

Important is here that the exponent $\theta$ also enters the auto-correlation $A(t)$. Actually the first numerical estimate of the exponent $\theta$ for the Ising model is from the measurement of $A(t)$ by taking $z$ as an input [4,5]. In this case, however, the error induced by $z$ is relatively big since the dynamic exponent $z$ is usually not known so rigorously and the ratio $d / z$ is much bigger than $\theta$. In contrast to this, with the exponent $\theta$ obtained from the initial increase of the magnetization, one can get a rather accurate value for the exponent $z$ from the autocorrelation. Compared with the traditional measurement of $z$ from the exponential decay of the auto-correlation in the long-time regime, to some extent, our short-time dynamic approach is free of critical slowing down. Due to the large time correlation length, for big lattice sizes the traditional measurement is very difficult since independent configurations can hardly be generated. However, in our short-time dynamic approach the measurement is always carried out in the short-time regime, where the system rather rapidly converges to the universal power law behaviour as the lattice size increases. In principle, we do not have the problem how to generate independent configurations efficiently. On the other hand, in the dynamic approach the average is really the sample average rather than the time average based on the ergodicity assumption. This fact may show its merit in future.

Here we should stress that the dynamic exponent $z$ is originally defined in the long-time regime of the dynamic evolution. It is a conceptual progress that we can determine it from the universal short-time behaviour. More importantly, not only the dynamic exponent $z$ but actually also the static exponents, which are defined in equilibrium state, can numerically be measured from the short-time dynamics, e.g. from the second moment $M^{(2)}(t)$.

To measure the auto-correlation $A(t)$ and the second moment $M^{(2)}(t)$, we have performed the simulation of $m_{0}=0$ with a lattice size $N=128$. In Fig. 2, $A(t)$ and $M^{(2)}(t)$ are plotted in double-log scale with the solid line and dotted line respectively. After a microscopic time scale $t_{m i c} \sim 50$, nice power law behaviour is observed. In the numerical simulations for the Ising model and Potts model, it is known that the microscopic time scale for the second moment is somehow longer than that for the magnetization [7]. This is also the case for the $\mathrm{SU}(2)$ lattice gauge theory. We have performed a power law fit in the time interval $[60,250]$ and obtain the exponent $\theta-d / z=-0.745(12)$ while $(d-2 \beta / \nu) / z=0.825(14)$. These results are consistent with those of the two-dimensional Ising model, $\theta-d / z=-0.737(01)$ and $(d-2 \beta / \nu) / z=0.817(07)$. Taking the exponent $\theta$ as an input, we can calculate the values for the dynamic exponent $z$ and the static exponent $\beta / \nu$. All these results and, for comparison

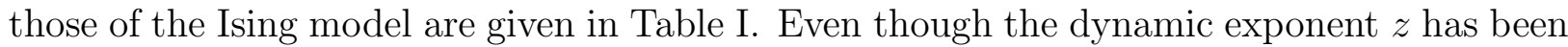
known for a long time, for the lattice gauge theory our measurement is the first reliable one. The measured value $\beta / \nu=0.120(18)$ is also in agreement with $\beta / \nu=0.120(08)$ obtained in reference [15], and with the exact value $\beta / \nu=0.125$ for the two-dimensional Ising model.

In conclusion, we have investigated the universal short-time behaviour of the $(2+1)$ dimensional dynamic SU(2) lattice gauge theory. The critical initial increase of the Polyakov loop is observed. The dynamic exponents $\theta$ and $z$ as well as the static critical exponent $\beta / \nu$ 
are determined from the power law behaviour of different observables at the early stage of the time evolution. The values are consistent within the errors with those of the two-dimensional Ising model. The results strongly support that there exists an universal short-time scaling behaviour for the dynamic $\mathrm{SU}(2)$ lattice gauge theory, and also suggest that the $(2+1)$ dimensional dynamic SU(2) lattice gauge theory and the two-dimensional Ising model are in the same dynamic universality class.

Acknowledgement: One of the authors (K.O) would like to acknowledge the hospitality of the Theory Group of The Elementary Particle Physics, University of California, Los Angeles for his stay for one year during when the paper has been completed. 


\section{REFERENCES}

[1] H. K. Janssen, B. Schaub and B. Schmittmann, Z. Phys. B 73, 539 (1989).

[2] Z.B. Li, U. Ritschel and B. Zheng, J. Phys. A: Math. Gen. 27, L837 (1994).

[3] L. Schülke and B. Zheng, Phys. Lett. A 204, 295 (1995).

[4] D. A. Huse, Phys. Rev. B 40, 304 (1989).

[5] K. Humayun and A. J. Bray, J. Phys. A 24, 1915 (1991).

[6] N. Menyhárd, J. Phys. A: Math. Gen. 27, 663 (1994).

[7] K. Okano, L. Schülke, K. Yamagishi and B. Zheng, Nucl. Phys. B 485, 727 (1997).

[8] K. Okano, L. Schülke, K. Yamagishi and B. Zheng, Monte Carlo simulation of the short-time behaviour of the dynamic XY model., Siegen Univ., 1997, preprint SI-97-02.

[9] P. Grassberger, Physica A 214, 547 (1995).

[10] P. Czerner and U. Ritschel, Phys. Rev. E 53, 3333 (1996).

[11] Z.B. Li, L. Schülke and B. Zheng, Phys. Rev. Lett. 74, 3396 (1995).

[12] Z.B. Li, L. Schülke and B. Zheng, Phys. Rev. E 53, 2940 (1996).

[13] P.C. Hohenberg and B.I. Halperin, Rev. Mod. Phys. 49, 435 (1977).

[14] B. Svetitsky and L.G. Yaffe, Nucl. Phys. B 210, 423 (1982).

[15] J. Christensen and P.H. Damgaard, Nucl. Phys. B 348, 226 (1991).

[16] M. Teper, Phys. Lett. B 313, 417 (1993).

[17] S.N. Majumdar, A.J. Bray, S. Cornell and C. Sir, Phys. Rev. Lett. 77, 3704 (1996).

[18] L. Schülke and B. Zheng, Monte Carlo Measurement of the Global Persistence Exponent., Siegen Univ., preprint, SI-97-03, 1997.

[19] H. K. Janssen, in Topics in Modern Statistical Physics, edited by G. Györgyi, I. Kondor, L. Sasvári and T. Tél (World Scientific, Singapore, 1992). 


\section{TABLES}

\begin{tabular}{|l|l|l|l|}
\hline \hline & $\theta$ & $\mathrm{z}$ & $\beta / \nu$ \\
\hline $\mathrm{SU}(2)$ & $0.192(02)$ & $2.135(27)$ & $0.120(18)$ \\
\hline Ising & $0.191(01)$ & $2.155(03)$ & 0.125 \\
\hline \hline
\end{tabular}

TABLE I. The exponents $\theta, z$ and $2 \beta / \nu$ measured from the short-time dynamics with the heat-bath algorithm. 


\section{FIGURES}

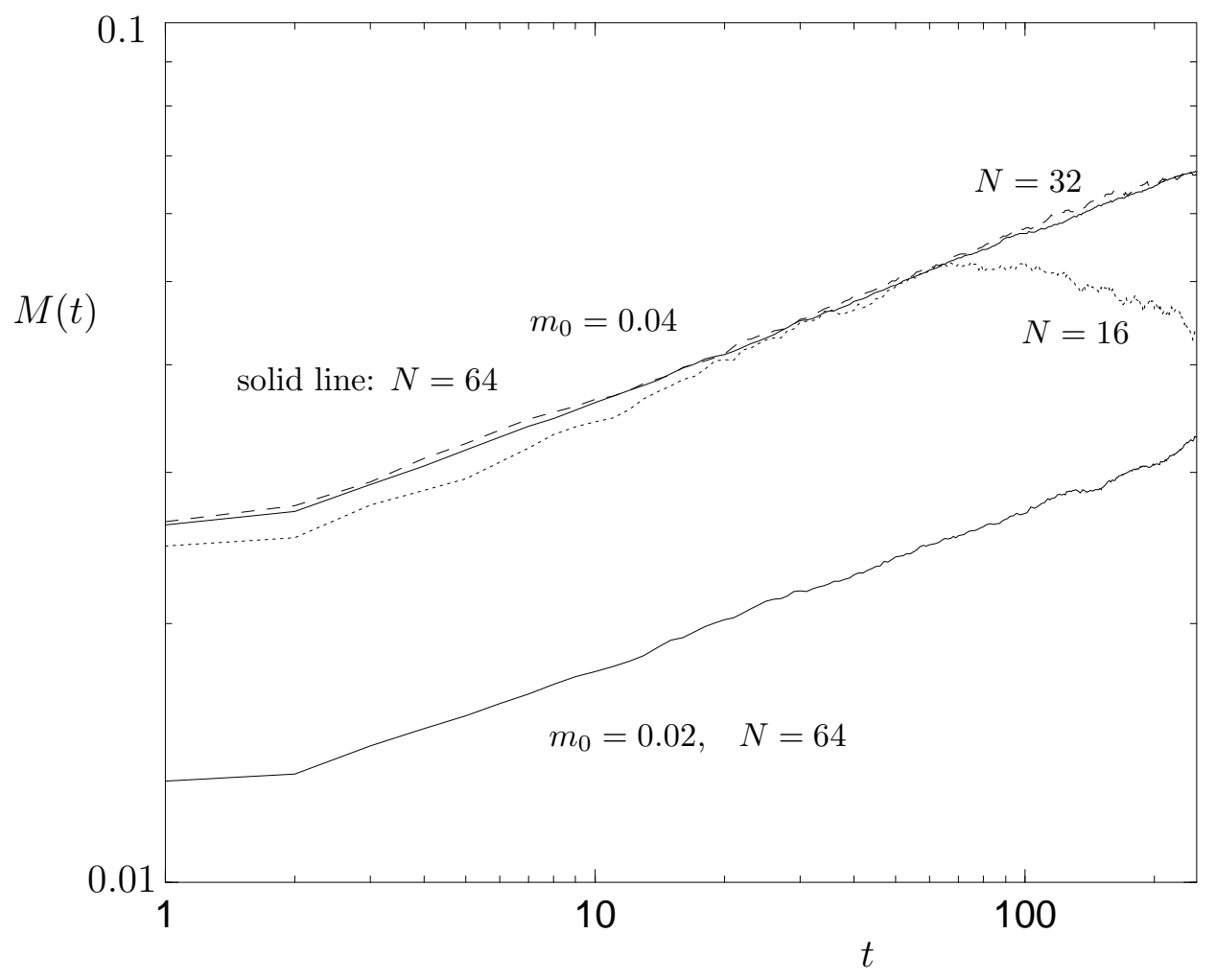

FIG. 1. The time evolution of the magnetization for different lattice sizes $N$ and initial magnetization $m_{0}$ with the heat-bath algorithm is plotted in double-log scale. 


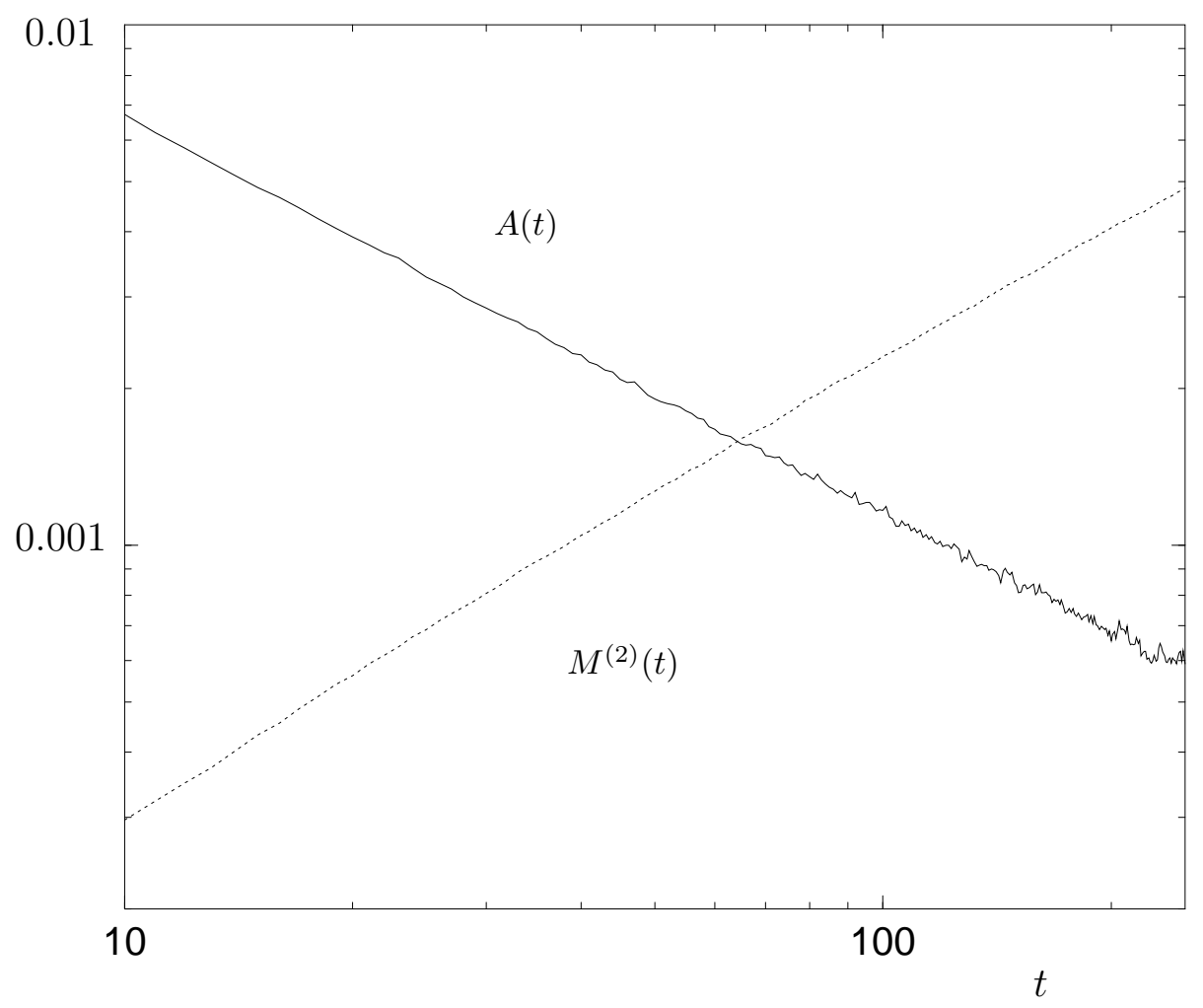

FIG. 2. The auto-correlation $A(t)$ and the second moment $M^{(2)}(t)$ for the lattice sizes $N=128$ and initial magnetization $m_{0}=0$ obtained with the heat-bath algorithm is plotted in double-log scale. 